\title{
Le rôle des lésions induites par les rayons ultraviolets dans l'initiation des cancers cutanés
}

Si les ultraviolets (LV) sont connus pour être des agents mutagènes dans de nombreux systèmes vivants ainsi que pour leur rôle étiologique dans la survenue des cancers cutanés, la démonstration moléculaire de la responsabilité des lésions induites sur l'ADN par les LV dans le développement des tumeurs de la peau n'est apparue que récemment, en particulier grâce à des études d'épidémiologie moléculaire ayant pour cible le gène suppresseur de tumeur $p 53$.

Le gène $p 53$ est le gène le plus fréquemment modifié dans les tumeurs humaines. De très nombreuses mutations, majoritairement des mutations faux sens, ont été caractérisées. Elles se regroupent principalement sur cinq régions du gène correspondant aux séquences conservées au cours de l'évolution. Ces mutations se répartissent différemment suivant les cancers et peuvent, dans certains cas, refléter la spécificité des carcinogènes physiques ou chimiques responsables de l'initiation tumorale $\left(m / s \quad n^{\circ} 3, \quad v o l .8\right.$, p. 289).

Le meilleur exemple d'association entre carcinogène physique et cancer est observé pour les tumeurs de la peau. En effet, il est clair que le spectre de mutation du gène $p 53$ décrit dans les tumeurs de la peau (carcinomes spino- et basocellulaires) diffère statistiquement de ceux décrits dans les autres cancers humains mais est, en revanche, très proche de ceux observés sur d'autres gènes cibles traités aux LV dans des systèmes modèles $[1,2]$. Ainsi, la plupart des mutations $p 53$ dans les tumeurs de la peau sont localisées au niveau de séquences bipyrimidiques qui sont des points chauds de lésions induites par les LV. Par ailleurs, ces mutations sont en majorité des transitions $C$ vers $\mathrm{T}$ avec une forte proportion de tran- sont la véritable signature des UV et qui correspondent probablement à la réplication translésionnelle de deux pyrimidines endommagées. Toutes ces caractéristiques sont exacerbées si l'on s'intéresse aux tumeurs de peau de patients atteints de xeroderma pigmentosum (XP), un syndrome humain héréditaire où l'absence de réparation des lésions induites par les UV est corrélée à une très haute fréquence de cancers sur les zones de la peau exposées au soleil [3]. Ainsi $100 \%$ des mutations du gène $p 53$ décrites dans les tumeurs de la peau de ces malades sont situées au niveau de séquences bipyrimidiques $(90 \%$ dans les tumeurs de peau d'individus normaux) et $55 \%$ sont des transitions en tandem CC vers TT (contre $14 \%$ dans les tumeurs de peau d'individus normaux). Cette répartition est statistiquement très différente de celle rapportée pour les tumeurs internes $[1,4]$. Il est surtout intéressant de noter que $95 \%$ des mutations p53 trouvées dans les tumeurs de malades XP sont dues à la présence de photolésions non réparées localisées sur le brin non transcrit du gène, alors que dans les tumeurs de peau non XP, les photolésions à l'origine des mutations sont localisées à peu près également sur les deux brins. Cette différence confirme que les processus de réparation des deux brins de l'ADN fonctionnent différemment. Elle montre aussi que, dans les cellules $\mathrm{XP}$ issues de patients très déficients dans la réparation de l'ADN (groupes de complémentation A et C), le très faible taux de réparation (inférieur à $10 \%$ du témoin) ne permet la réparation que du brin transcrit par l'ARN polymérase II, laissant sur le brin non transcrit des lésions qui vont donner naissance, après réplication, à des mutations localisées au niveau de séquences bipyrimidiques situées sur le brin non transcrit. Le spectre de mutation trouvé dans les tumeurs de peau de patients XP est ainsi le premier exemple de réparation préférentielle dans des tissus humains in vivo [4].

Les résultats d'épidémiologie moléculaire obtenus dans les tumeurs de la peau en utilisant les oncogènes de la famille ras [5] et, surtout, le gène $p 53$ mettent donc clairement en évidence le rôle des lésions induites par les UV dans les cancers de la peau chez l'homme. Enfin, notre étude confirme l'intérêt du gène $p 53$ pour corréler un spectre de mutation à un agent endommageant l'ADN de façon spécifique. Cela est dû au nombre important de sites mutables sur ce gène (environ 300) ainsi qu'au rôle fondamental du produit de ce gène dans le maintien du cycle cellulaire normal et dans l'initiation de la cancérogenèse.

A.S.

1. Dumaz N, StaryA, Soussi T, DayaGrosjean I, Sarasin A. Can we predict solar ultraviolet radiation as a causal event in human tumors by analysing the mutation spectra of the $p 53$ gene. Mutat Res 1994 (sous presse).

2. Ziegler A, Leffell DJ, Kunala S, Sharma HW, Gailani M, Simaon JA, Baden HP, Shapiro PE, Bale AE, Brash DE. Mutation hot spots due to sunlight in the $p 53$ gene of nonmelanoma skin cancers. Proc Nall Acad Si USA 1993; 90 : 4216-20.

3. Sarasin A. Les gènes humains de la réparation de l'ADN. médecine/sciences $1994 ; 10$ : 43-54.

4. Dumaz N, Drougard C, Sarasin A, DayaGrosjean L. Specific UV-induced mutation spectrum in the $p 53$ gene of skin tumors from DNA repair deficient Xeroderma pigmentosum patients. Proc Natl Acad Sa USA $1993 ; 90: 10529-33$.

5. Daya-Cirosjean I, Robert L, Drougard C, Suarez HC; Sarasin A. High mutation frequency in ras genes of skin tumors isolated from DNA-repair deficient Xeroderma pigmentosum patients. Cancer Res 1993; 53 : 1625-9. 